\title{
EFFECTS OF ACTIVATED CARBON PROPERTIES ON THE ADSORPTION OF NAPHTHALENE FROM AQUEOUS SOLUTIONS
}

CO Ania*, B Cabal, C Pevida, A Arenillas, JB Parra, F Rubiera, JJ Pis

Instituto Nacional del Carbón, CSIC. Apartado 73, 33080 Oviedo (Spain)

*Corresponding author E-mail: conchi@incar.csic.es

\begin{abstract}
The aim of this work was to investigate the role of the porous and chemical heterogeneities of activated carbons in the adsorption of naphthalene from aqueous media. A commercially available activated carbon was used as adsorbent, and its surface heterogeneity was systematically altered by heat treatment at 450 and $850{ }^{\circ} \mathrm{C}$, obtaining a series of carbons with various oxygen contents and similar surface functionalities. The results confirmed that the adsorption of naphthalene strongly depends on the pore size distribution of the adsorbent, particularly narrow microporosity. Moreover, oxygen functionalities reduced the accessibility and affinity of naphthalene to the inner pore structure via formation of hydration clusters. Consequently, the hydrophobic/hydrophilic character of the adsorbent is important, since it dominates the competitive adsorption of water. Adsorbents with a high non-polar character (i.e., low oxygen content) have proven to be more efficient for naphthalene adsorption.
\end{abstract}

Keywords: PAH, adsorption, liquid phase, activated carbon 


\section{INTRODUCTION}

The coking process generates typically between 0.4 and $4 \mathrm{~m}^{3}$ of wastewater per tonne of coke, containing a wide variety of pollutants in extremely high concentrations (i.e., phenols, cyanides, thiocyanate, hydrocarbons). Wastewater also contains polycyclic aromatic hydrocarbons (PAHs) at significant concentrations (up to $30 \mathrm{mg} \mathrm{L}^{-1}$ ), which produce an effluent with a high COD (chemical oxygen demand) and a low BOD (biological oxygen demand). These compounds are considered as priority pollutants $[1,2]$ as they are long-term persistent and highly mobile throughout the environment (semi-volatiles). Most of them also present carcinogenic properties, and their presence in water media becomes a serious environmental problem due to diffusion into groundwater reservoirs and drinking water. Besides, they are often discharged by industrial activities to urban streams that will be later treated in wastewatertreatment plants. In such cases these compounds pose an additional technological problem due to their low biodegradability, PAHs cannot be easily destroyed by biological treatment and many of them can inhibit the biological process. Activated carbon, however, is able to deal with almost any organic compound present in the waste. Moreover, adsorption on activated carbons presents several advantages such as being a low-energy demanding process, the possibility of regeneration, and a broad availability of adsorbents. All of this makes the adsorption of PAH from liquid phase on activated carbons an interesting field of research.

Although extensive research has been carried out on the application of activated carbons in environmental remediation [3], and particularly on the adsorption of organic compounds and metallic pollutants, until now, most of the research on pollution by PAH has been performed in the gas phase [4], while investigation on aqueous streams has remained a little behind [5-7].

The main objective of this research is to investigate the mechanism of retention of naphthalene on activated carbons, and to elucidate the role of porosity and surface composition of the adsorbent. For this purpose, the surface chemistry of an activated carbon was carefully modified via heat treatment, so as to obtain a series of adsorbents with various oxygen contents and similar porous structure. As opposed to chemical modification of a carbon material by wet oxidation, where different functionalities are randomly introduced in the carbon matrix, in this 
case the nature of the surface functionalities can be better controlled by the temperature of the heat treatment.

\section{EXPERIMENTAL}

\section{Materials}

A commercial activated carbon, $\mathrm{NC}$, obtained from chemical activation of wood with phosphoric acid and supplied by Norit was chosen for this study. The selected carbon was treated under a nitrogen flow rate of $100 \mathrm{~mL} \mathrm{~min}{ }^{-1}$, at the temperatures of 450 and $850{ }^{\circ} \mathrm{C}$ during 30 min. The samples will be denoted as NC450 and NC850, respectively. The samples were ground, sieved and a particle size fraction of $0.71-1 \mathrm{~mm}$ was used.

\section{Adsorption from solution}

Adsorption of naphthalene was carried out at room temperature in a stirred batch system as described elsewhere [8]. In brief, different amounts of carbons (from $50 \mathrm{mg}$ to $1 \mathrm{~g}$ ) were weighed and added to 15 bottles containing $100 \mathrm{~mL}$ of the hydrocarbon aqueous solution with an initial concentration of $30 \mathrm{ppm}$. The covered bottles were allowed to shake for $72 \mathrm{~h}$ at a constant temperature. Kinetic studies revealed that the adsorption equilibrium was established after 3 days. After equilibration the concentration in the solution was determined using an UV-Vis spectrophotometer at the corresponding wavelength $(275.5 \mathrm{~nm})$. The amount adsorbed of solute per unit gram of adsorbent, $q_{e}$, was evaluated as $q_{e}=\left(C_{o}-C_{e}\right) * V / m$, where $C_{o}$ is the initial concentration, $C_{e}$ the remaining concentration after equilibrium, $V$ is the volume of the solution and $m$ the mass of the adsorbent.

\section{Textural and chemical characterization}

Textural characterization was carried out by measuring the $\mathrm{N}_{2}$ (Micromeritics ASAP 2010M) and $\mathrm{CO}_{2}$ (Micromeritics TriStar) adsorption isotherms at -196 and $0{ }^{\circ} \mathrm{C}$, respectively. Before the experiments, the samples were outgassed under vacuum at $120^{\circ} \mathrm{C}$ overnight. The $\mathrm{N}_{2}$ isotherms were used to calculate the specific surface area, $\mathrm{S}_{\mathrm{BET}}$, total pore volume, $\mathrm{V}_{\mathrm{T}}$, and pore size distributions which were evaluated using density functional theory (DFT). The distribution of pores smaller than $0.7 \mathrm{~nm}$ (narrow microporosity) was assessed from $\mathrm{CO}_{2}$ adsorption isotherms 
[9] by applying the Dubinin-Stoeckli (DS) equation [10] and using $1.023 \mathrm{~g} \mathrm{~cm}^{-3}$ as the density of adsorbed $\mathrm{CO}_{2}$ and 0.36 as $\beta$ parameter.

\section{Thermal analysis}

Thermal analysis was carried out using a TGA92 thermogravimetric analyzer from Setaram (TG). Temperature programmed desorption (TPD) experiments were carried out under an argon flow rate of $50 \mathrm{~cm}^{3} \mathrm{~min}^{-1}$, at a heating rate of $15^{\circ} \mathrm{C} \mathrm{min}^{-1}$, up to a final temperature of $1000{ }^{\circ} \mathrm{C}$. An Omnistar quadrupole mass spectrometer from Balzers (MS), and a Nexus infrared spectrometer from Nicolet (FTIR), linked to the thermobalance were used for evolved gas analysis. To avoid secondary reactions, a probe was placed very close to the sample crucible of the thermobalance in the direction of the gas flow. The gas lines between the TG and the MS/FTIR were heated to $180^{\circ} \mathrm{C}$ in order to avoid cold points. For each experiment, about 35 $\mathrm{mg}$ of a ground carbon sample was used.

\section{RESULTS AND DISCUSSION}

\section{Textural and chemical characterization of the as-received samples}

Detailed characteristics of the pore structure of the series of heat-treated carbons obtained from gas adsorption data are presented in Table 1, along with the variation on the oxygen content and the point of zero charge $\left(\mathrm{pH}_{\mathrm{PZC}}\right)$. The parent carbon presented a type IV nitrogen adsorption isotherm and a hysteresis loop at relative pressures above 0.5 , which is in agreement with a well-developed mesoporosity obtained from the DFT analysis. Heating at $450{ }^{\circ} \mathrm{C}$ did not alter the porosity of the sample and the volume of micropores remained unchanged. However, the amount of oxygen considerably decreased (Table 1), although the nature of the carbon remained still acidic, as inferred from the $\mathrm{pH}_{\mathrm{PZC}}$ of the sample. Thus, after applying a low temperature heat treatment an adsorbent with unchanged porous texture but lower amount of oxygencontaining groups was obtained.

On the other hand, the NC850 sample presented a loss in the porosity, particularly concerning the mesopores volume and the microporosity determined by $\mathrm{N}_{2}$ adsorption (i.e., micropores of larger sizes). The narrow microporosity evaluated from the $\mathrm{CO}_{2}$ data shows a slight increase, suggesting an effect of porosity opening, likely as a result of partial gasification of the sample 
due to evolution of volatiles upon heating. At the same time there is a drastic reduction in the oxygen content (almost $70 \%)$.

The changes in surface chemistry pointed out by the oxygen content, as well as the nature of the evolved functionalities were investigated by TPD-MS/FTIR (Figure 1). The TPD results include the quantification of the $\mathrm{CO}$ and $\mathrm{CO}_{2}$ evolved in an argon atmosphere. Small amounts of $\mathrm{CO}_{2}$ and $\mathrm{CO}$ were desorbed during the TPD experiments for the NC850 sample, indicating that the majority of the oxygenated groups had already been evolved during the former treatment at $850^{\circ} \mathrm{C}$, which is in agreement with the oxygen content evaluated by elemental analysis. The value of the $\mathrm{pH}_{\mathrm{PZC}}$ of $\mathrm{NC} 850$ indicates that the few groups remaining are of a basic nature.

On the other hand, for the sample treated at $450{ }^{\circ} \mathrm{C}, \mathrm{NC} 450$, desorption of $\mathrm{CO}_{2}$ at low temperature decreased in comparison with $\mathrm{NC}$, as a consequence of surface groups having been eliminated during the heat treatment, whereas the profiles of $\mathrm{CO}_{2}$ and $\mathrm{CO}$ at high temperature are similar to those of the parent carbon. It should be underlined that despite a loss of $40 \%$ in the oxygen content, the functionalities are still of an acidic nature, as reflected by its $\mathrm{pH}_{\mathrm{PZC}}$ value.

Summarizing, it is possible to modulate the characteristics of the activated carbons by a gradual heat treatment of the parent carbon, obtaining a series of samples with precisely the same porous texture and almost two times less oxygen. This will allow us to get a proper understanding of the mechanism of naphthalene adsorption from aqueous medium on activated carbons.

\section{Adsorption of naphthalene from liquid phase}

Figure 2 illustrates the experimental naphthalene adsorption isotherms of the samples. From their shape it is seen that a marked plateau appears at low doses of adsorbent, indicating the achievement of the saturation limit. A detailed analysis of the plots of adsorption curves leads to the observation that isotherms of $\mathrm{NC} 450$ and $\mathrm{NC} 850$ intersect at low solution concentration, suggesting two regimes of adsorption. Below the intersection point (large adsorbent dose), adsorption followed the trend $\mathrm{NC}<\mathrm{NC} 450<\mathrm{NC} 850$. Therefore the effect of the changes on the surface properties of the adsorbents depends on the range of equilibrium concentration 
considered in the isotherms. Similar observation has been reported on aromatic compounds [11].

Taking into account the non-polar nature of the adsorbate, this behavior should be related to the hydrophobic/hydrophilic behavior of the carbons or, in other words, to a strong solvent competition. It seems that there is a preferential adsorption of naphthalene over water in NC850 sample (confirmed by the L-type adsorption isotherm), whereas a non-negligible competition of water is present in carbons with high oxygen contents, NC450 and NC (i.e., hydrophilic nature). In these two cases, the presence of oxygenated sites on the surface of the carbon pores drastically changed the adsorption behavior. The isotherms presented a somewhat S-type at low solution concentration where little adsorption occurs and increases progressively as the concentration is raised. This suggests a strong competitive adsorption of water, and naphthalene adsorption is enhanced likely through naphthalene-naphthalene interactions at higher concentrations. The oxygen groups are ideal sites for water adsorption, favoring the creation of water clusters at the entrance of the pores. Those hydration clusters might effectively reduce the accessibility and affinity observed of the naphthalene molecules to the inner pore structure [12]. For NC850, its hydrophobic character minimizes the solvent competition, but it presented a lower porosity (Table 1), which explains the decrease in the uptake when the active sites of the carbon are filled (high concentrations).

It is well known that the porosity of carbons is a critical factor in adsorption processes of organic compounds both in gas or liquid phase. Consequently, the adsorption behavior must be investigated taking into account the textural changes induced in the carbons during heat treatment.

It is evident from the results in Table 1 that $\mathrm{NC}$ and $\mathrm{NC} 450$ have the same porosity (no apparent changes in either pore volumes or pore size distributions). Despite this fact, their performance towards naphthalene adsorption is extremely different. The decrease in the oxygen content of NC450 with regards to NC resulted in an important increase in naphthalene uptake over the whole concentration range (parallel isotherms). This demonstrates that, as expected, naphthalene adsorption is enhanced by adsorbents of a hydrophobic nature. 
Besides, this is an important finding since one might expect that adsorption of a non-polar compound should be exclusively linked to the porosity of the carbons. However, this assumption does not explain why the two carbons with identical porous network displayed so different naphthalene removal. To clarify this issue and investigate the exact mechanism of naphthalene adsorption as well as the role of porosity, the nature of these interactions between the adsorbate and the adsorbents was explored by thermogravimetric analysis of the exhausted samples.

DTG curves of the carbons before and after naphthalene exposure are shown in Figure 3. A common feature in all cases is a peak at temperatures lower than $100{ }^{\circ} \mathrm{C}$, due to the removal of physisorbed water. In the case of NC and NC450 after naphthalene adsorption, the peaks at temperatures higher than $700{ }^{\circ} \mathrm{C}$, are linked to the decomposition of the surface functionalities, since they were also observed in the profiles of the carbons before naphthalene adsorption.

After naphthalene exposure, a peak of high intensity and centered at around $400{ }^{\circ} \mathrm{C}$ was observed for all the samples. In the case of NC sat and NC450 sat the peak overlaps with the one from the decomposition of surface groups which is displayed in the profiles before adsorption although after naphthalene adsorption the intensity of the peak substantially increases, for which it was attributed to the evolution of naphthalene physisorbed in the micropores. This fact was corroborated by the identification of the evolved gases by TG-FTIR (Figure 4).

For both NC sat and NC450 sat, naphthalene evolves at the same temperature, which points out that the same adsorption forces are involved in the process of adsorption. This is in good agreement with the assumption that the active sites are the micropores, and that the adsorption proceeds via physisorption of naphthalene on the microporosity. In the case of NC850 sat, the peak shifted to a higher temperature, which is also consistent with this premise. As it has been mentioned above, heating at $850{ }^{\circ} \mathrm{C}$ turned out in a decrease of medium-size micropores and mesopores, at expenses of an increase in the narrow microporosity. This demonstrates that the narrow micropores whose dimensions match those of the adsorbate are active sites of high adsorption potential. 
The analysis of the gases evolved from TPD experiments by FTIR (cf. Figure 4) confirmed the assignment of the desorption peak to naphthalene. The fact that the naphthalene desorption peak detected by FTIR, appears at temperatures higher than those shown by the DTG curves is due to the condensation of naphthalene in cold points at the interface between the TG and FTIR apparatus. Moreover, it can be inferred that naphthalene does not undergo decomposition reactions while heating and it is desorbed in a molecular state, since no other hydrocarbons were detected.

Thus we can infer that retention proceeds via dispersive interactions between the $\pi$-electron density of the polyaromatic structure of the adsorbate and the electron-rich region in the graphene layers (these occurring in the micropores). However, to explain the enhancement in loading capacity of NC450 vs NC, despite their similar porosity, the exact role of the oxygen functionalities must be clarified.

It is well-known that the oxygen functionalities are mainly located at the edges of the basal planes and the entrance of the pores in activated carbons [13]. Taking this into account and being the majority of surface groups of electron-acceptor character, functionalization of the carbons withdraws $\pi$-electron density of the graphene layers. At the same time, the functionalization of the carbon also increases its polar character which, as it has been discussed before, enhances the formation of water clusters that hinder the adsorption of naphthalene. Consequently, with the removal of oxygen, regions of higher $\pi$-electron densities (where the dispersive interactions are favored) appear, and more naphthalene molecules cover the carbon surface.

Support for the beneficial effect of microporosity in the adsorption of naphthalene was provided by the analysis of the porosity of the samples after naphthalene adsorption by means of $\mathrm{N}_{2}$ and $\mathrm{CO}_{2}$ adsorption isotherms (cf. Table 1). The most drastic effect was observed in NC850 sample, particularly in the volume of narrow micropores that was reduced by $66 \%$ after adsorption. The main difference, as a result of naphthalene adsorption in this sample was the decrease in the 
volume of pores narrower than $1 \mathrm{~nm}$ (Figure 5), indicating that naphthalene is mainly retained therein.

These results confirmed that the adsorption of naphthalene depends greatly on the pore size distribution of the adsorbent, being enhanced in those pores of narrower dimensions.

\section{CONCLUSIONS}

The adsorption of a non-polar polyaromatic pollutant on activated carbons with different surface and textural heterogeneities was explored, and the results confirmed that the adsorption of naphthalene strongly depends on the pore size distribution of the adsorbent, particularly narrow microporosity. The study of the textural properties of the carbons after the adsorption of naphthalene revealed that the main difference as a consequence of retention is a decrease in the volume of pores narrower than $1 \mathrm{~nm}$.

However, the adsorption behavior should not be exclusively linked to the porosity of the carbons, since two carbons with identical porous network and different oxygen functionalities displayed very different naphthalene removal. This can only be explained by assuming that oxygen functionalities reduce the accessibility and affinity of naphthalene molecules to the inner pore structure via formation of hydration clusters. Consequently, the hydrophobic/hydrophilic character of the adsorbent is important, since it dominates the undesirable competitive adsorption of water. Adsorbents with a high non-polar character (i.e., low oxygen content) have proven to be more efficient for naphthalene adsorption.

\section{Acknowledgements}

Work carried out with a financial grant from the European Coal and Steel Community (Project 7220-PR139). COA thanks the Spanish MEC for a postdoctoral fellowship (MEC EX20040612).

\section{References}

[1] Directive 2000/60/EC, EU Water Framework Directive. Official Journal L 327, on 22 December 2000. 
[2] Directive 96/62/EC, EU Air Quality Framework Directive. Official Journal L 309, on 27 November 2001.

[3] Activated carbon surfaces in environmental remediation. (Ed. T.J. Bandosz), Interface Science and Technology, vol 7, Elsevier, New York, 2006.

[4] A.M. Mastral, T. García, R. Murillo, M.S. Callén, J.M. López, M.V. Navarro, Ind. Eng. Chem. Res. 42 (2003) 155.

[5] C.O. Ania, B. Cabal, C. Pevida, A. Arenillas, J.B. Parra, F. Rubiera, J.J. Pis, Water Research (submitted)

[6] M. Seredych, A.Gierak, Colloids Surfaces A: Physicochem. Eng. Aspects 245 (2004) 61.

[7] M. Seredych, V.M. Gun'ko, A. Gierak, Appl. Surf. Sci. 242 (2005) 154.

[8] C.O. Ania, T.J. Bandosz, Langmuir 21 (2005) 7752.

[9] D. Lozano-Castello, D. Cazorla-Amoros, A. Linares-Solano, Carbon 42 (2004) 1233.

[10] M.M. Dubinin, H.F. Stoeckli. J. Colloid Interf. Sci., 75 (1980) 34.

[11] A.P. Terzyk. J. Colloid Interf. Sci. 268 (2003) 301.

[12] M. Franz , H.A. Arafat, N.G. Pinto, Carbon 38 (2000) 1807.

[13] J.B. Donnet, Carbon 20 (1982) 267. 


\section{Figure Captions}

Figure 1. TPD-MS desorption rate under argon of the as-received samples.

Figure 2. Equilibrium adsorption isotherms of naphthalene on the studied carbons. Inset: linear scale.

Figure 3. DTG of the carbons before and after (sat) naphthalene adsorption.

Figure 4. TPD-FTIR of the samples after naphthalene exposure.

Figure 5. Micropore size distributions obtained by the DRS method applied to the $\mathrm{CO}_{2}$ adsorption isotherms of the carbons before (open symbols) and after (closed symbols) naphthalene exposure.

\section{Table Captions}

Table 1. Chemical and textural features of the studied carbons before and after (sat) the adsorption of naphthalene from aqueous solutions 
Figure 1.
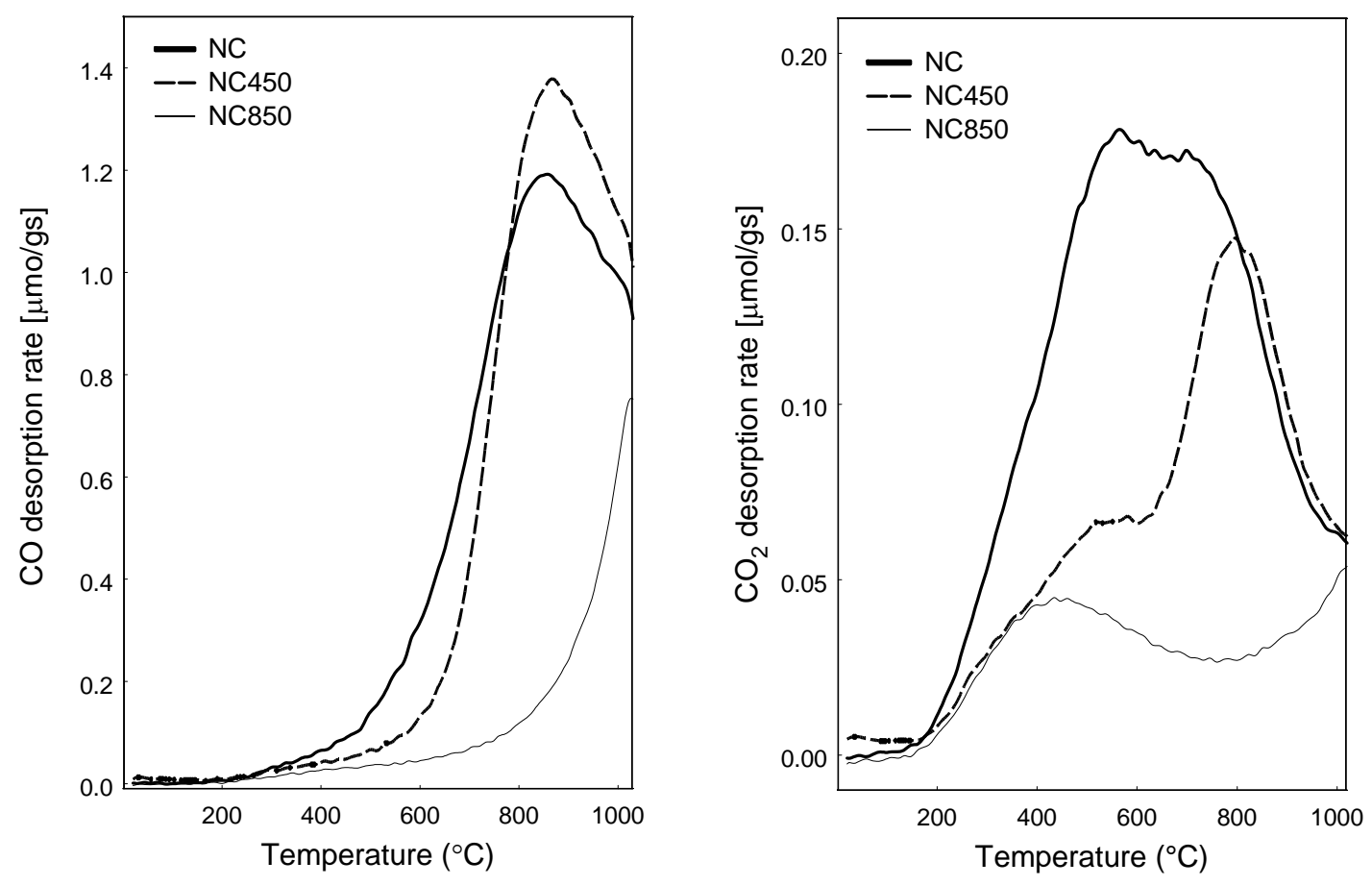
Figure 2.

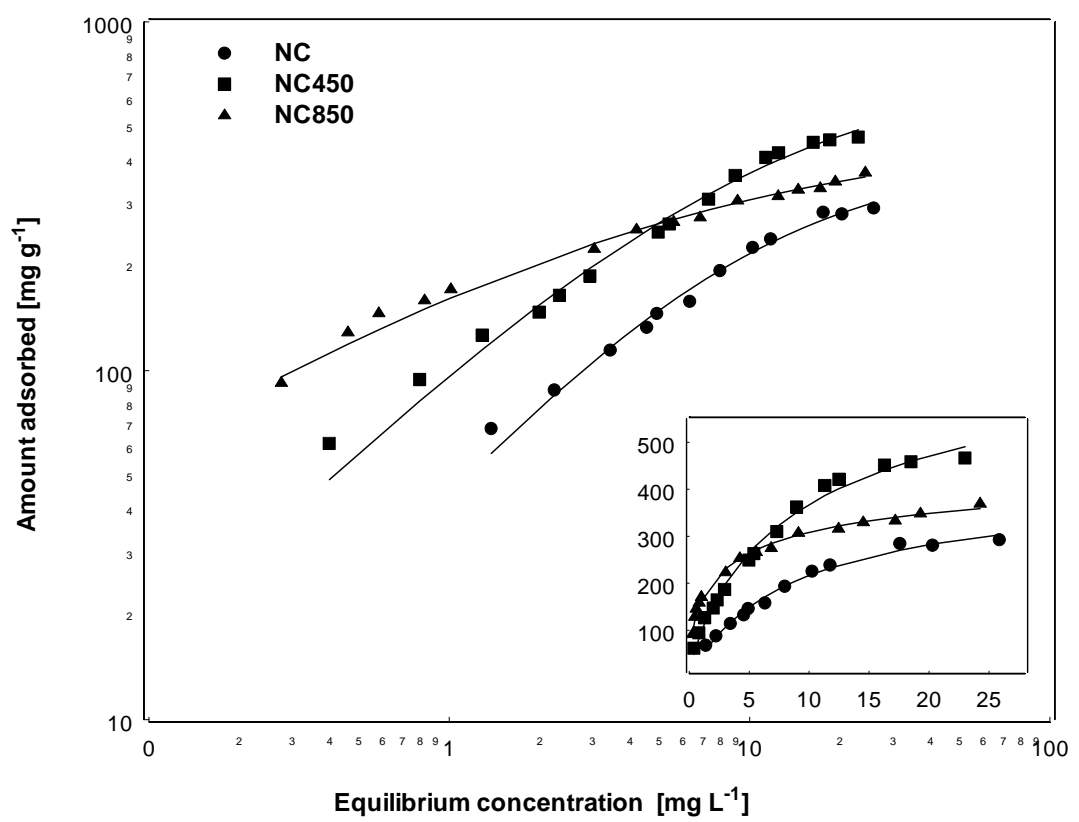


Figure 3.
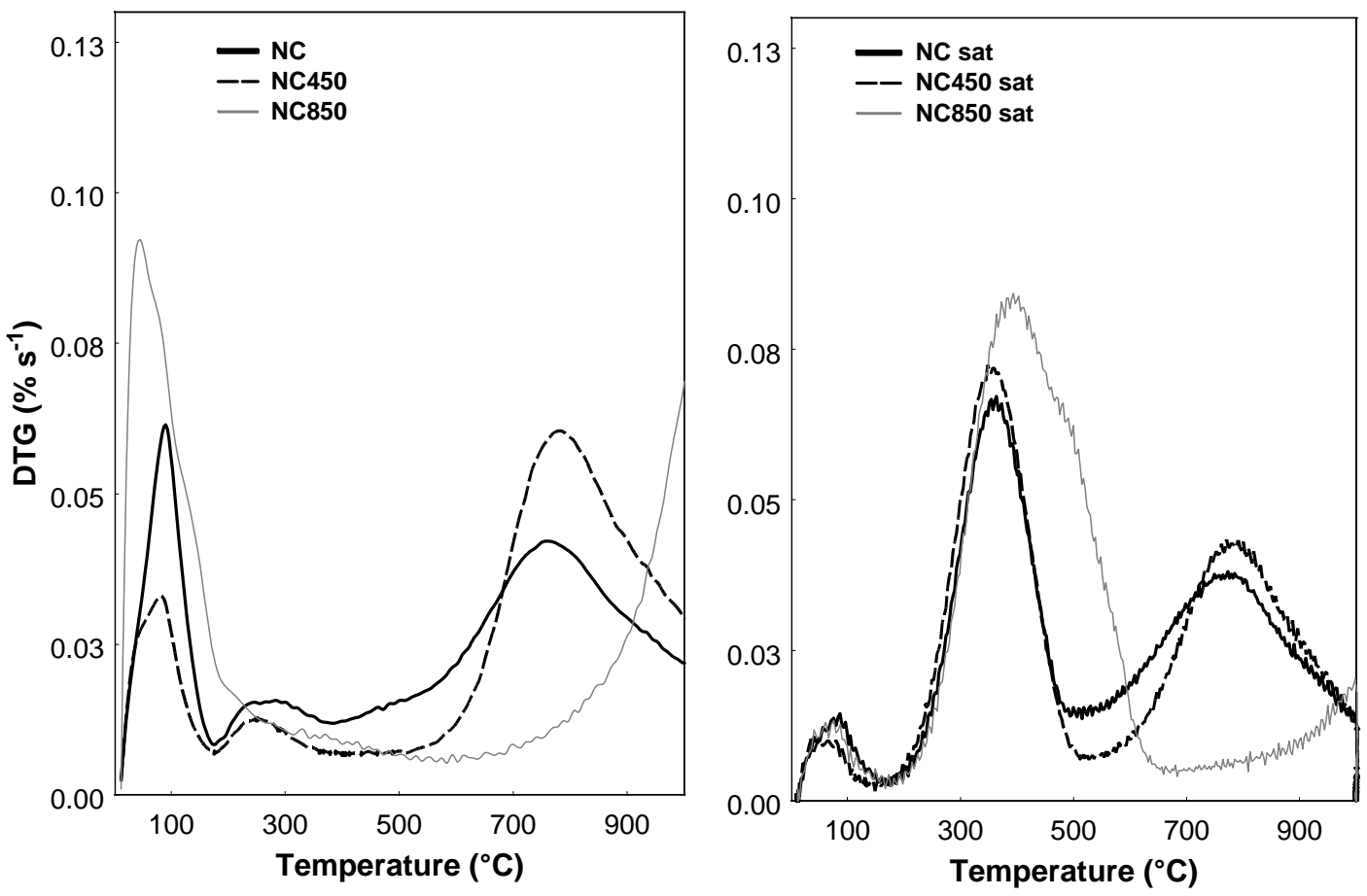
Figure 4.

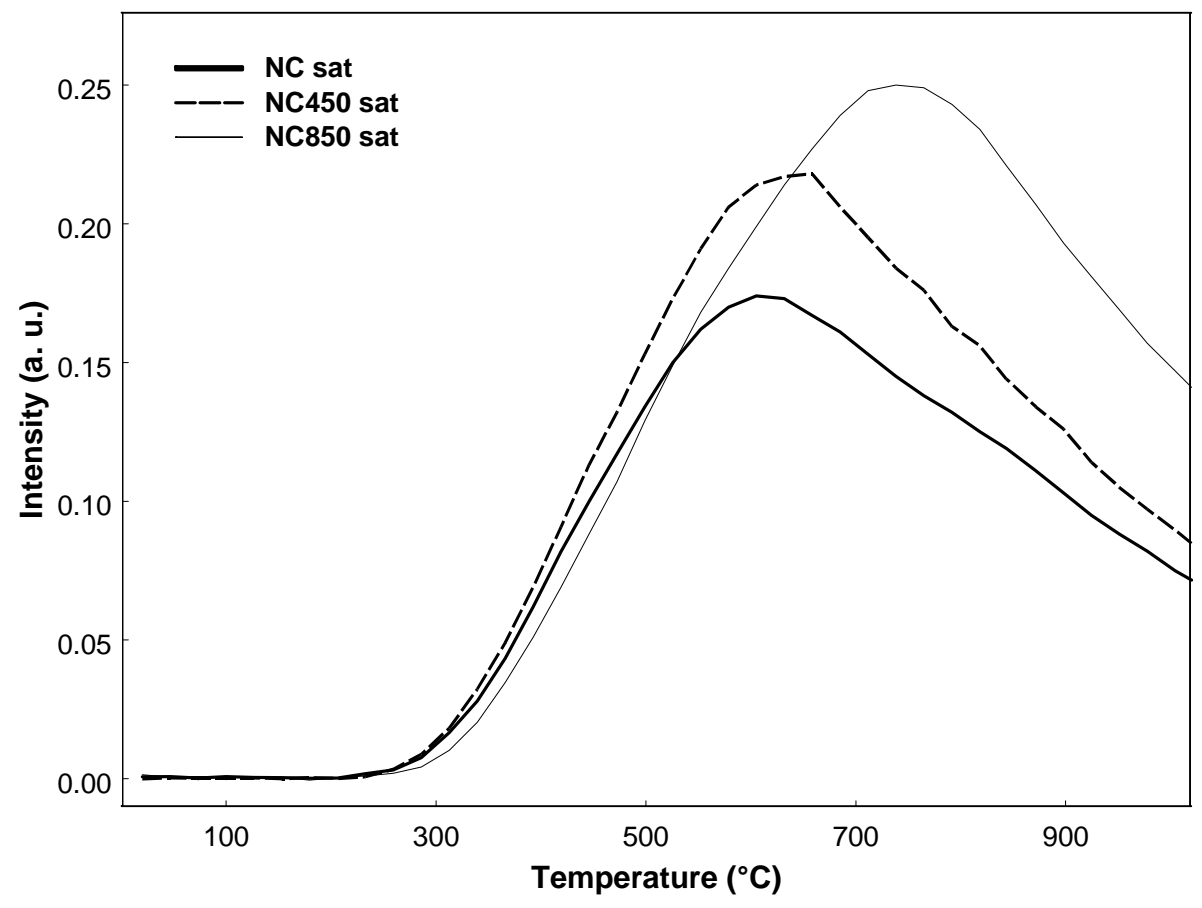


Figure 5.

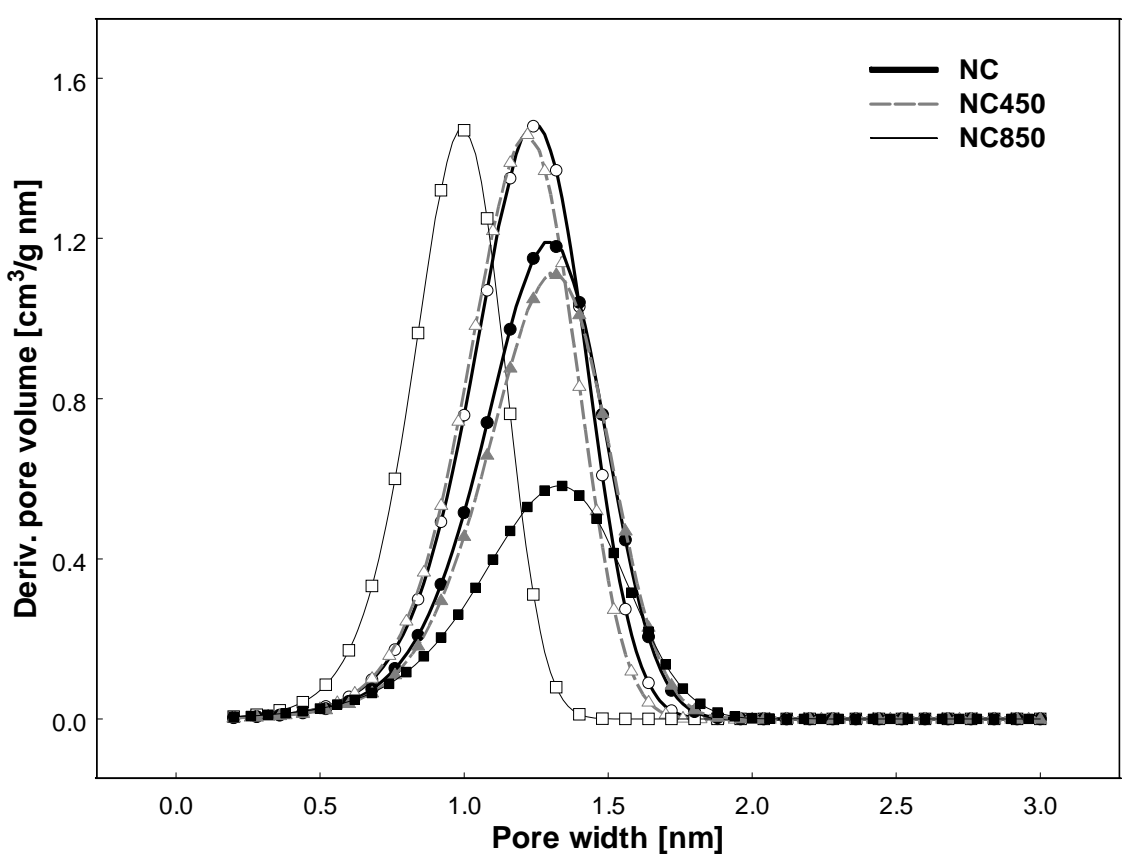


Table 1.

\begin{tabular}{|c|c|c|c|c|c|c|}
\hline & $\begin{array}{c}\text { O } \\
\text { [wt. \%] }\end{array}$ & $\begin{array}{l}\text { pH } \\
\text { PZC }\end{array}$ & $\begin{array}{c}\mathrm{S}_{\mathrm{BET}} \\
{\left[\mathrm{m}^{2} \mathbf{g}^{-1}\right]}\end{array}$ & $\begin{array}{c}\text { V MICROPORES }^{\mathrm{a}} \\
{\left[\mathrm{cm}^{3} \mathbf{g}^{-1}\right]}\end{array}$ & $\begin{array}{c}\text { V }_{\text {MESOPORES }}{ }^{\mathrm{a}} \\
{\left[\mathrm{cm}^{3} \mathbf{g}^{-1}\right]}\end{array}$ & $\begin{array}{c}\mathrm{V}_{\mathrm{CO}}^{\mathrm{b}} \\
{\left[\mathrm{cm}^{3} \mathrm{~g}^{-1}\right]}\end{array}$ \\
\hline NC & 12.7 & 3.1 & 1477 & 0.403 & 0.374 & 0.181 \\
\hline NC450 & 7.6 & 3.5 & 1489 & 0.408 & 0.372 & 0.171 \\
\hline NC850 & 3.9 & 7.8 & 1063 & 0.300 & 0.239 & 0.188 \\
\hline NC sat & - & - & 1137 & 0.348 & 0.292 & 0.136 \\
\hline NC450 sat & - & - & 1165 & 0.326 & 0.302 & 0.126 \\
\hline NC850 sat & - & - & 566 & 0.190 & 0.142 & 0.063 \\
\hline
\end{tabular}

${ }^{a}$ evaluated from the DFT method applied to the $\mathrm{N}_{2}$ adsorption isotherms at $-196^{\circ} \mathrm{C}$

${ }^{\mathrm{b}}$ evaluated from the DR method applied to the $\mathrm{CO}_{2}$ adsorption isotherms at $0{ }^{\circ} \mathrm{C}$ 